\title{
Defect Motion in Rotating Fluids
}

\author{
Juan Millán-Rodríguez, ${ }^{1}$ Michael Bestehorn, ${ }^{1, *}$ Carlos Pérez-García, ${ }^{1}$ Rudolf Friedrich, ${ }^{2}$ and Marc Neufeld ${ }^{2}$ \\ ${ }^{1}$ Departamento de Física y Matemática Aplicada, Facultad de Ciencias, Universidad de Navarra, 31080 Pamplona, Navarra, Spain \\ ${ }^{2}$ Institut für Theoretische Physik und Synergetik der Universität Stuttgart, Pfaffenwaldring 57/4, 70550 Stuttgart, Germany
}

(Received 19 January 1994)

\begin{abstract}
Defect motion in pattern formation (gliding and climbing, defects annihilation, pinning effects, and pattern reorientation) is analyzed for the first time when a fluid layer heated from below rotates slowly around its vertical axis. This system allows for a continuous change from a (approximately) variational problem (no rotation) to a nonvariational one by increasing the externally applied rotation rate. By a numerical integration of the 3D-hydrodynamic equations, climbing and gliding velocities of defects are determined. A mechanism leading to a reorientation of a parallel roll structure is discussed.
\end{abstract}

PACS numbers: 47.20.-k, 47.11.+j, 47.32.- $\mathrm{y}$

Patterns in spatially extended systems far from equilibrium usually present a large number of defects. They appear spontaneously as a consequence of the intrinsic dynamics or due to sidewall effects, unavoidable in experiments [1]. In this Letter we wish to study RayleighBénard (RB) convection under rotation. The interest for instabilities of this system has received a renewed attention on the experimental [2-5] as well as on the theoretical field [6-10]. In contrast to previous work we wish to analyze here pattern formation in slowly rotating fluids well below the Küppers-Lortz instability (KLI) [11]. It is well known that in this regime perfect patterns of parallel rolls are stable, but we show that the presence of defects may lead to qualitative changes in the spatiotemporal dynamics of pattern formation even at the onset of convection.

In $\mathrm{RB}$ convection without rotation the pattern is formed by rolls, and the usual defects are dislocations (point defect) and grain boundaries (line defect). The texture can be described by $\Psi(\mathbf{r})=a(\mathbf{r}) \cos [\mathbf{k} \cdot \mathbf{r}+\phi(\mathbf{r})]$, where $\Psi$ is a convective variable (the vertical velocity or the temperature), $a$ and $\phi$ are the slowly varying amplitude and phase, respectively, and $\mathbf{k}$ denotes the wave vector. At the core of a defect, $a$ vanishes. The circulation $C$ of $\nabla \phi$ over a closed line including the defect is equal to $2 \pi q$, where $q$ is an integer and defined as the topologic charge of the defect. (Defects with a charge different from \pm 1 are not observed because they are unstable.)

Theoretical [12-17] and experimental [18-21] studies of defect motion in a macroscopic structure show that the defect usually displaces along the rolls (climbing). In variational systems this can be explained by the selection of an "optimal" wavelength due to defect motion and finds its analogy in the Peach-Köhler force in solid state physics [13].

When rotation is added to $\mathrm{RB}$ convection, the Coriolis force influences drastically the pattern dynamics [10], and defects play an important role in the transition to spatiotemporal complexity. Moreover, the system loses its variational character even at the threshold of convection which may lead to inherently time dependent structures. Numerical studies based on extended SwiftHohenberg equations [6-8] gave indications that, even in the case of a small rotation rate, new effects may appear as a consequence of the interaction between defects and the whole pattern.

Evolution equations for the problem can be found in Ref. [22]. Here we shall restrict our study to fluids with a large Prandtl number. The usual decomposition of the velocity field into a poloidal $\left(\nabla \times \nabla \times\left[A(\mathbf{r}, t) \mathbf{z}_{0}\right]\right)$ and a toroidal $\left(\nabla \times\left[B(\mathbf{r}, t) \mathbf{z}_{0}\right]\right)$ part leads to the basic equations for the three coupled scalar functions $A, B$, and the temperature $\Theta$ :

$$
\begin{aligned}
\operatorname{Ta}_{z} \Delta_{2} A(\mathbf{r}, t)+\Delta_{2} B(\mathbf{r}, t) & =0 \\
\Delta^{2} \Delta_{2} A(\mathbf{r}, t)-\operatorname{Ta}_{z} \Delta_{2} B(\mathbf{r}, t)-R \Delta_{2} \Theta(\mathbf{r}, t) & =0, \\
\Delta_{2} A(\mathbf{r}, t)+\left(\partial_{t}-\Delta\right) \Theta(\mathbf{r}, t) & =-\boldsymbol{v}(\mathbf{r}, t) \cdot \nabla \Theta(\mathbf{r}, t),
\end{aligned}
$$

with $\Delta_{2}=\partial_{x x}+\partial_{y y}$ and $\Delta=\Delta_{2}+\partial_{z z}$. Control parameters are the Rayleigh number $R$, proportional to the vertically applied temperature gradient, and the Taylor number Ta $=2 \Omega d^{2} / v$, proportional to the angular velocity $\Omega$ of the fluid layer.

To integrate numerically Eq. (1) under realistic, no-slip boundary conditions on the top and the bottom of the fluid, we use an algorithm developed earlier by one of us (M. B.) for 3D convection in binary mixtures [23]. That algorithm was 
improved further and applied successfully to convection under Bénard-Marangoni conditions in Ref. [24] where details of the numerical code can be found.

Here we wish to test our numerical algorithm first in the case of a fluid layer at rest $($ Ta $=0)$, by studying the experimentally obtained defect dynamics of Ref. [19]. Afterwards we proceed to the case of rotation. We investigate the evolution of a pair of dislocations created by the initial condition (Fig. 1):

$$
\Theta(\mathbf{r}, t=0)=a \sin (k x) \cos (\pi z), \quad k= \begin{cases}k_{1} & \text { for } 0<y<L_{y} / 4, \quad 3 L_{y} / 4<y<L_{y}, \\ k_{2} & \text { for } L_{y} / 4<y<3 L_{y} / 4,\end{cases}
$$

where $L_{x}, L_{y}$ is the length and width of the layer and $k_{2}-k_{1}=2 \pi / L_{x}$.

To obtain comparable results with experiments we used aspect ratios $L_{x}: L_{y}: \lambda_{c} / 2=38: 21: 1$, where $\lambda_{c} / 2=$ $\pi / k_{c}$ is the diameter of the linearly fastest growing rolls. As initial values we took $k_{1}=k_{c}, k_{2}=(20 / 19) k_{c}$ as in Ref. [19]. The numerical resolution was 6 mesh points per roll in lateral and 16 points in the vertical direction, resulting in a grid of $228 \times 126 \times 16$ points. With a symmetric initial condition as (2) defect motion is parallel to the roll structure (climbing).

We have evaluated the climbing velocity $V_{\mathrm{CL}}$ for different values of $\varepsilon=\left(R-R_{c}\right) / R_{c}$. After an initial transient phase that is roughly proportional to $1 / \varepsilon, V_{\mathrm{CL}}$ reaches a constant value [Fig. 2(a)], as long as the separation of the two defects is large enough that they can be regarded as being isolated. [We measured the velocity only at certain times during the defect motion, namely when the defect crosses a mesh point in the $y$ direction. This, and not the numerical inaccuracy, is the reason for the discontinuities of the velocities during the transient phases shown in Fig. 2(a). However, we are not interested in the transients but rather in the final constant values of the velocities.] We adopted the parameters of [19] (silicon oil with kinetic viscosity of $5 \mathrm{cSt}$ at $25^{\circ} \mathrm{C}$, thermal diffusivity $\kappa=7 \times 10^{-4} \mathrm{~cm}^{2} / \mathrm{s}$ corresponding to a Prandtl number of $\operatorname{Pr}=70$; depth of the layer $d=$

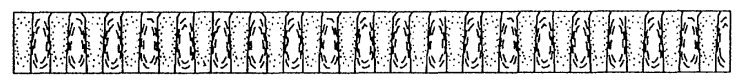

VERTICAL PLANE: $y=L / 2$

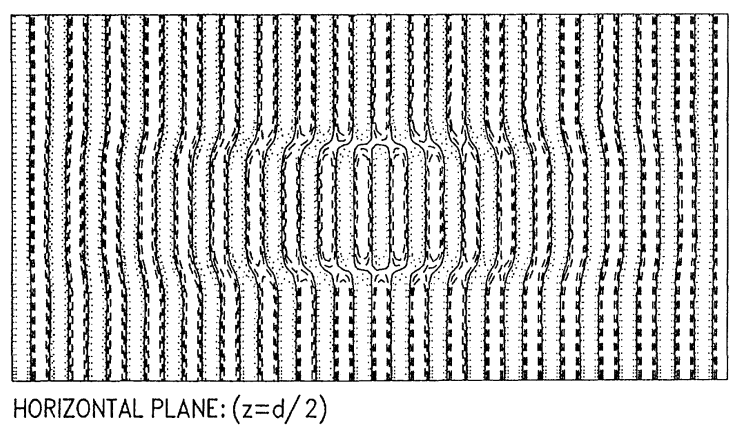

FIG. 1. Initial condition for $\Theta$ in the horizontal plane at $z=d / 2$ (bottom) and in the vertical plane at $y=L_{y} / 2$ (top). Two defects are created by the inclusion of an extra roll in the center of the texture.
$2.56 \mathrm{~mm}$; vertical thermal diffusion time $\tau=93 \mathrm{~s}$ ). For quantitative comparison with experiments, the velocities found in our numerical simulations are dimensioned with $\kappa / d=9.84 \mathrm{~cm} / \mathrm{h}$.

Along the theory developed for variational systems [13], the climbing velocity $V_{\mathrm{CL}}$ is $V_{\mathrm{CL}} \propto D_{\perp}^{3 / 2}$, where $D_{\perp}$ is the diffusion coefficient of a phase disturbance perpendicular to the rolls. From Ref. [25] it is known that $D_{\perp}$ depends on the Prandtl number Pr as well as on $\varepsilon$ and thus in lowest order of $\varepsilon$

$$
V_{\mathrm{CL}}=\gamma\left(\frac{\delta k}{k_{c}}+\alpha(\operatorname{Pr}) \varepsilon+O\left(\varepsilon^{2}\right)\right)^{3 / 2},
$$

with $\delta k$ being the difference between the wave number of the pattern and the critical one and $\alpha=0.015(\operatorname{Pr}=\infty)$ or $\alpha=0.046(\operatorname{Pr}=70)$. For $\gamma$ we take the value of the literature $[12,13,25] \gamma=53$, which is in reasonable agreement with the experimental results [Fig. 2(b), solid line] [19]. We found quantitative agreement between the relation (3) and our numerical simulations [Fig. 2(b), dashed line], at least for smaller $\varepsilon<1$. We note that Eq. (3) is the leading part of an $\varepsilon$ expansion and therefore only valid near the onset.

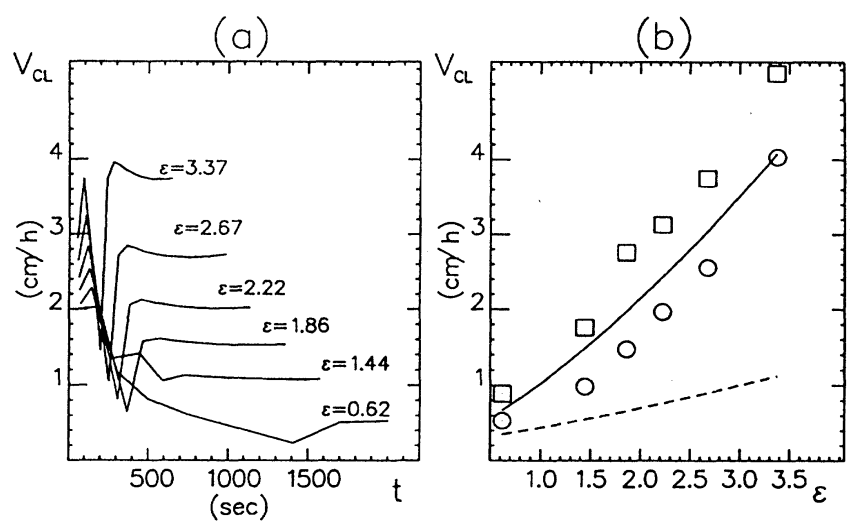

FIG. 2. A comparison between the climbing velocity in the experiment of Pocheau et al. [19] and our numerical results without rotation $(\mathrm{Ta}=0)$. To obtain dimensionalized quantities, we used material properties of [19] (cf. text). (a) Temporal evolution of climb velocities. They saturate after the initial phase of pattern formation that is roughly $\propto 1 / \varepsilon$. (b) The dependence of $V_{\mathrm{CL}}$ on $\varepsilon$ can be fitted by the expression $V_{\mathrm{CL}}=\gamma\left(\delta k / k_{c}+\alpha \varepsilon\right)^{3 / 2}$ (see text). Squares: data from [19], $\operatorname{Pr}=70$; circles: our results for infinite $\operatorname{Pr}$. 

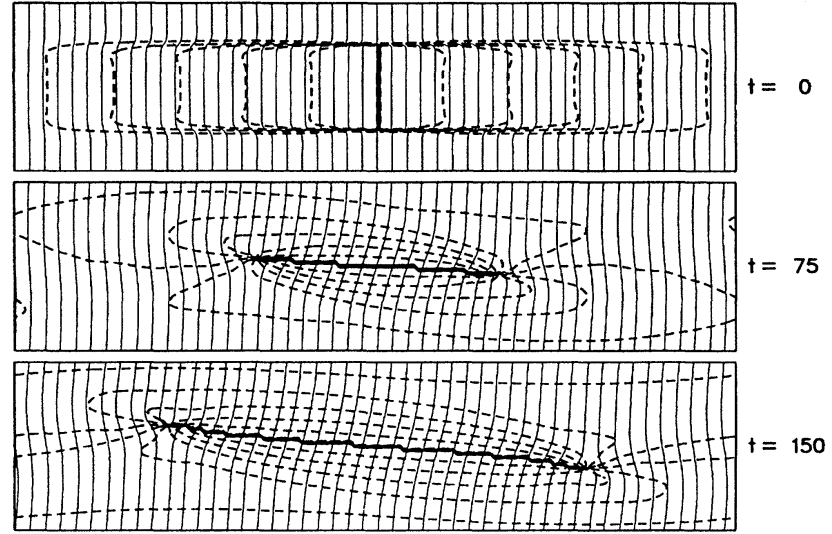

FIG. 3. Gliding of a pair of oppositely charged defects under the influence of a Coriolis force $(\mathrm{Ta}=-10)$ due to a clockwise rotation of the layer near threshold of convection $(\varepsilon=0.15)$. Dashed lines: contour lines of the phase $\Phi$, defined as $\Theta=$ $a \sin (k x+\Phi)$. Solid lines: zero values of $\Theta$. The two defects are linked by a phase discontinuity of $2 \pi$ (bold line). View on the horizontal plane. Here and in the following figures the time unit is the vertical diffusion time $\tau$.

Now we study the influence of rotation $(\mathrm{Ta}>0)$ on the initial pattern (2) well below KLI, which occurs for $\mathrm{Ta}_{\mathrm{KLI}}= \pm 54.8$. Since we expect a gliding of defects we use a more elongated geometry with aspect ratios $L_{x}$ : $L_{y}: \lambda_{c} / 2=48: 12: 1$. The rather large extension in the $x$ direction allows the defects to separate well. Since we are now interested in smaller $\varepsilon$ values we use a somewhat lower spacial resolution of $192 \times 48 \times 12$ points. After a short initial transient the defects begin to travel mainly perpendicular to the rolls (gliding). Rotation favors the lateral displacement of a dislocation. Defects carrying opposite charge travel in the opposite lateral direction with the same velocity $V_{\mathrm{GL}}$ (Fig. 3). Near the onset we found a linear dependence of $V_{\mathrm{GL}}$ on $\varepsilon$ and Ta (Fig. 4) of $V_{\mathrm{GL}} \propto q \varepsilon \mathrm{Ta}$, in accordance with our recent analytical studies [26].

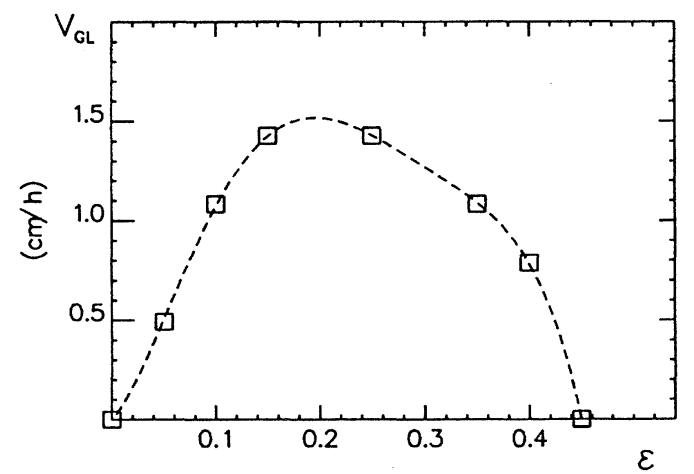

FIG. 4. Glide velocity of a defect for $\mathrm{Ta}=-10$. Above a certain value for $\varepsilon$, damping due to pinning effects increases faster then the driving force and $V_{\mathrm{GL}}$ decreases again. For $\varepsilon>0.45$, the defect is pinned by a roll and stops. The dashed line is a polynomial fit and thought of as a guide to the eye.
As the lateral boundary conditions are periodic, defects may circulate and interact until they finally annihilate by collision (Fig. 5). Another interesting feature is also obvious from this figure: The motion and annihilation of a pair of oppositely charged defects give rise to an eventual reorientation of parallel rolls in the sense of the externally applied rotation (the same behavior can be seen in the evolution in Fig. 3). Note that this effect occurs below KLI and is not related with the reorientation of about $60^{\circ}$ found there. This effect has not been observed yet in experiments, it is completely new. The experiments of Ref. [5] were performed for larger $\mathrm{Ta} \approx$ 30 , and the transitions reported there are clearly of the KLI type where pattern dynamics is not mainly mediated by defects as in our case. Zhong and Ecke argue that the decrease of the KLI point occurs due to the cylindrical symmetry and finite size effects of their cell. Our own numerical computations showed that KLI occurs always above $\mathrm{Ta}_{\mathrm{KLI}}$ if periodic lateral boundary conditions are applied.

From the theory for variational models a decrease of the glide mobility is expected for rather large values of $\varepsilon$ due to the increase of the potential barrier caused by the small

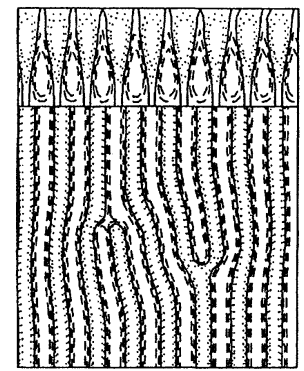

$T=3$

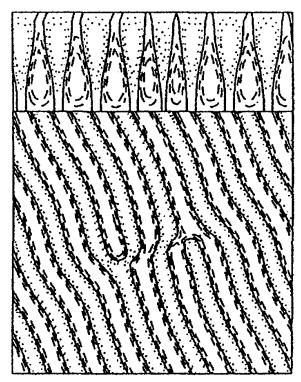

$\mathrm{T}=45$

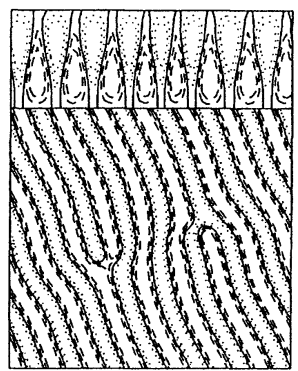

$T=44$

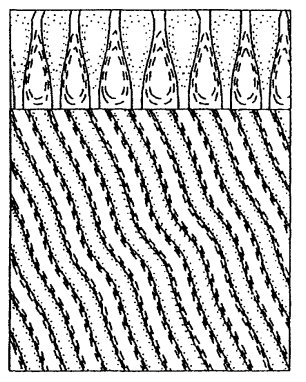

$T=46$
FIG. 5. Oppositely charged defects can eventually disappear by a collision. After the annihilation, a pattern of parallel rolls with changed orientation remains. Here the external rotation is counterclockwise. Note that the cell is periodically continued like a torus and the defects may travel around the cell. $\quad(\mathrm{Ta}=$ 22, $\varepsilon=1.0$.) As in Fig. 1, the stripe on the top shows a side view, whereas the square below gives the horizontal view of the entire cell at $z=d / 2$. 


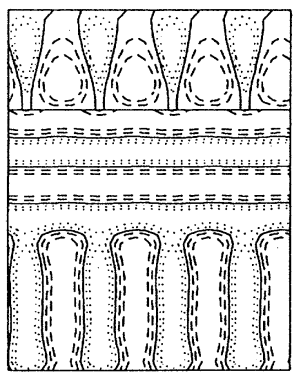

$T=0.1$

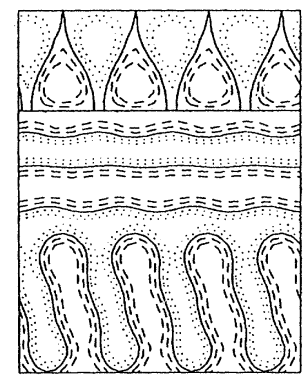

$T=6$
FIG. 6. Gliding of roll edges at a grain boundary provides another mechanism for a global reorientation of convection rolls. Same view as in Fig. 5. ( $\mathrm{Ta}=22, \varepsilon=1.5$.) The external rotation is counterclockwise.

scale roll structure [27]. The defect tends to be pinned [28,29]. We found the same behavior: The pinning force tries to damp the defect motion. For small $R$, the driving force created by rotation may overcome the damping and the defect glides with a velocity that first increases with $R$. For larger $R, V_{\mathrm{GL}}$ decreases again and finally reaches zero (Fig. 4), the defect is trapped by a roll.

From our numerical studies it turned out that the climb motion is much slower than the glide motion, at least in the range of $\varepsilon$ that was the subject of our studies. Because of defect separation perpendicular to the rolls caused by rotation, the wavelengths above and below each defect may adjust themselves to the same value and the driving force in the roll direction cancels.

Finally, we wish to study the influence of rotation on the dynamics of a grain boundary (GB). This was investigated theoretically [30] and experimentally [31] in $\mathrm{RB}$ convection without rotation. Here we introduce a GB as an initial condition, just by taking two domains with perpendicular rolls. When we apply rotation to the cell, the edge of the rolls begins to glide, eventually leading to a parallel roll pattern. The first few time steps of that transient evolution are shown in Fig. 6. In a cylindrical vessel, a similar gliding velocity of the defects along the sidewalls is observed. These gliding motions push the whole pattern to rotate at constant velocity as has been observed in recent experiments [5] and obtained in theoretical works [6-9].

We acknowledge partial financial support from DGICYT (Spanish Government) under Grant No. PB93-0708.

*To whom correspondence should be addressed.

[1] M. C. Cross and P.C. Hohenberg, Rev. Mod. Phys. 65, 851 (1993), and references therein.
[2] J. J. Niemela and R. J. Donnelly, Phys. Rev. Lett. 57, 2524 (1986).

[3] E. Bodenschatz, D. S. Cannell, J.R. de Bruyn, R. Ecke, Y.C. Hu, K. Lerman, and G. Ahlers, Physica (Amsterdam) 61D, 77 (1992).

[4] F. Zhong, R. Ecke, and V. Steinberg, J. Fluid Mech. 249, 135 (1992).

[5] F. Zhong and R. Ecke, Chaos 2, 163 (1992).

[6] J. Millán-Rodríguez, C. Pérez-García, M. Bestehorn, M. Fantz, and R. Friedrich, Phys. Rev. A 46, 4729 (1992).

[7] M. Fantz, R. Friedrich, M. Bestehorn, and H. Haken, Physica (Amsterdam) 61D, 147 (1992).

[8] M. Neufeld, R. Friedrich, and H. Haken, Z. Phys. 92, 243 (1993).

[9] E. Y. Kuo and M.C. Cross, Phys. Rev. E 47, R2245 (1993).

[10] Y. Tu and M.C. Cross, Phys. Rev. Lett. 69, 2515 (1992).

[11] G. Küppers and D. Lortz, J. Fluid Mech. 35, 609 (1969).

[12] E.D. Siggia and A. Zippelius, Phys. Rev. A 24, 1036 (1981).

[13] Y. Pomeau, S. Zaleski, and P. Manneville, Phys. Rev. A 27, 2710 (1983).

[14] E. Dubois-Violette, E. Guazelli, and J. Prost, Philos. Mag. A48, 727 (1983).

[15] G. Tesauro and M.C. Cross, Phys. Rev. A 34, 1363 (1986).

[16] E. Bodenschatz, W. Pesch, and L. Kramer, Physica (Amsterdam) 32D, 135 (1988).

[17] J. D. Rodriguez and L. M. Pismen, Phys. Rev. A 44, 7980 (1991).

[18] V. Croquette and A. Pocheau, in Cellular Structures in Instabilities, edited by J.E. Wesfreid and S. Zaleski (Springer, Berlin, 1984).

[19] A. Pocheau and V. Croquette, J. Phys. 45, 35 (1984).

[20] S. Nasuno, S. Takeuchi, and Y. Sawada, Phys. Rev. A 40, 3457 (1989).

[21] S. Rasenat, V. Steinberg, and I. Rehberg, Phys. Rev. A 42, 5998 (1990).

[22] S. Chandrasekhar, Hydrodynamic and Hydromagnetic Stability (Clarendon, Oxford, 1961).

[23] M. Bestehorn, Physica (Amsterdam) 61D, 59 (1993).

[24] M. Bestehorn, Phys. Rev. E 48, 3622 (1993).

[25] P. Manneville and J. M. Piquemal, Phys. Rev. A 28, 1774 (1983).

[26] J. Millán-Rodríguez, C. Pérez-García, M. Bestehorn, M. Neufeld, and R. Friedrich, Chaos 4, 369 (1994).

[27] B. Malomed, A. A. Nepomnyashchy, and M. I. Tribelsky, Phys. Rev. A 42, 7244 (1990).

[28] Y. Pomeau, Physica (Amsterdam) 23D, 3 (1986).

[29] D. Bensimon, B. I. Shraiman, and V. Croquette, Phys. Rev. A 38, 5461 (1988).

[30] P. Manneville and Y. Pomeau, Philos. Mag. A48, 607 (1983).

[31] V. Croquette, M. Mory, and F. Schosseler, J. Phys. 44, 293 (1983). 